\title{
Ethical Controversy in Embryonic Stem Cell Research and Discussions on Public Policies
}

\author{
Chun Li \\ College of Life Sciences and Bioengineering, \\ School of Science \\ Beijing Jiaotong University \\ Beijing, China \\ betteryu@126.com
}

\author{
Honggang $\mathrm{Hu}^{*}$ \\ College of Life Sciences and Bioengineering, \\ School of Science \\ Beijing Jiaotong University \\ Beijing, China \\ hghu@bjtu.edu.cn
}

\author{
Lingling Hou \\ College of Life Sciences and Bioengineering, \\ School of Science \\ Beijing Jiaotong University \\ Beijing, China \\ llhou@bjtu.edu.cn
}

\begin{abstract}
With the rapid development of embryonic stem cell research, ethical issues arising from the research have increasingly become the focus of controversy. The main issues include controversy over moral status of embryos and protection of rights for embryo donors. This paper discusses the two major controversial issues arising in biomedical studies of stem cells, explores the directions and approaches to solve some of these issues and introduces the views on the corresponding policy formation.
\end{abstract}

Keywords-ethics; human stem cell research; embryo; donor; public policies

\section{INTRODUCTION}

Embryonic stem cell research is an important achievement of rapid development in modern life science and biomedicine and it has become a research highlight in this area. Embryonic stem cells (ESCs) are highly undifferentiated pluripotent cells derived from the primitive embryo before gastrula stage (5 7 days after fertilization), which can self-renew and differentiate into all kind of tissues and organs in the body. In 1998, American scientists successfully derived human embryonic stem cells (hESCs) from human blastocysts (Embryo 4 5 days), which can proliferate indefinitely in an undifferentiated state in vitro $[1,2]$. Due to its totipotent characteristics in development, hESC can differentiate into over 200 kinds of cells all over the body, such as trophoblast cells, nerve cells, hematopoietic cells and myocardial cells in the appropriate conditions and construct all tissues and organs in the body. So hESC shows very prominent advantage in the field of regenerative medicine. This technique is likely to change the present situation of basic research in human development, transplantation technology, cell and gene therapy, drug screening and other biomedical fields completely, and has promising prospects in scientific and clinical applications. In 2005, French scientists successfully used the ESCs of mice to repair the damaged cardiac muscle of sheep. This result indicates that it will become possible to use hES cells in treatment of heart disease [3]. The studies of the relationship between stem cells and a variety of complex diseases can help researchers to further explore the complex development course of diseases and the reasons for development of pathological cells without normal function. This is unachievable only with animal experiments. In addition, researchers can test the safety and effectiveness of new targeted drugs without human test through deriving disease-specific hESCs and drug intervention of their in vitro tissue culture.

However, the application of hESC involves a series of ethical issues impacting the traditional moral values, so hESC research has aroused huge ethical controversy. In recent years, domestic and foreign scholars have given great attention to the ethical issues in the development of hESC research. The ethical issues arising in hESC research mainly include: disposal of early embryos and how to correctly deal with embryo donors.

With rapid development of research in this aspect, it is very necessary and urgent to discuss the ethical issues caused by the research results. It is the common responsibility of scholars in life ethics to analyze the causes, find out the solutions and promote the formulation and improvement of the relevant policies.

\section{ETHICAL ISSUES IN STEM CELL RESEARCH AND ClinicAl APPLICATIONS}

\section{A. Ethical Conservancy over Embryos}

Due to the current level of technology, the derivation of human embryonic stem cells for research or medical purpose will inevitably cause damage to embryos. This involves protection of rights for early embryos and is one of the major controversial issues among ethicists and the public. In fact, whether early embryos have the same moral 
status as human beings is highly related to people's perception on these issues under different social, religious and cultural backgrounds. American bioethicist Engelhardt has proposed the "culture war" theory on embryonic stem cell research. He believes that the perception on the meaning of life and death varies with different cultures in the world, and the cultural disputes generated will profoundly affect the development of life ethics.

People who advocate embryo protection are largely from religious circles. Some religious persons resolutely oppose embryonic stem cell research. they believe that formation of a fertilized egg is the beginning of human life and simultaneously it has the innate moral status which remains unchanged in the whole process from gestation, birth to death. Before implanted into the uterus, all embryos have equal moral status as human beings, and even very small cell aggregates should not be violated regardless whether they are in a test tube or womb. However, not all religions give embryos the moral status equal to human beings. According to Judaism, Islam, Hinduism and many Western Christian traditions, embryos will not have the moral status until late pregnancy period. This perspective is more consistent with the analysis of moral status from the point of the rational function. In embryology, it is generally believed that after 14 days development, a fertilized egg will start differentiation into tissue and organ, and cells will have some obvious structure. That is to say, before this stage an embryo is just a structureless cell mass and has no sense or perception, and it is less likely to have the moral status as people. Therefore, the use of embryos less than 14 days old for research and therapy is acceptable.

Some believe that although a preimplantation embryo has not yet become a person, it has the potential to develop into a person, so destruction of such a potential means to deprive the right of the embryo's survival, and this cannot be accepted morally. Some scientists, who support embryonic stem cell research, believe that not all preimplantation embryos have the potential to develop into a human being, many IVF embryos can't successfully reach the stage of pregnancy, but the stem cells can be obtained from them. Unless they are implanted into the uterus, these preimplantation embryos will never develop into a complete human life.

In China, people's perception on embryos is greatly different from that of the Westerners. A survey indicated, most physicians in China believe that early embryos have no complete moral status, and they agree to apply stem cell research in clinical applications, in order to save the patients who suffer from incurable diseases. This is because China is less influenced by religions, its cultural diversification degree is lower than that of the Western countries, and the Chinese people have a rather consistent viewpoint on embryos. According to Chinese traditional morality and Confucianism, embryos can develop into human beings, but early embryos do not have the moral status, therefore, early embryos may enjoy a certain dignity but can't be considered equal to human, and the behavior of destroying the preimplantation embryos for therapy purpose is acceptable in China.
Although it is acceptable to derive stem cells from embryos for research and therapy purposes, we should still have a kind of respect for embryos and reverence for life. We should be cautious in the use of embryos. Embryos for research and therapy purpose should be surplus embryos from IVF or dead embryos. Deliberately creating embryos for research purpose will lead to the pure use of embryos as tools and for human cloning. The value of deliberately created embryos is not to develop them into human beings, but to use them as tools and they will be destroyed in the process. Although embryos don't have the moral status of human, this kind of research projects should be strictly controlled to avoid the abuse of embryos and development toward human cloning. These research projects should be also comprehensively assessed whether they have enough value. A report from the UK Department of Health states that stringent laws should be enacted to prevent created embryos from developing into cloning. For example, artificial embryos should be prohibited from being implanted into the uterus, and preimplantation embryos can't survive in vitro for more than 14 days [4].

In order to evade embryonic ethical issues of stem cells, scientists throughout the world make efforts unremitting in recent years. ACT claims they can obtain stem cells without destroying embryos. They derived single cell from human embryo consisting of 8 to 10 cells and cultured it into stem cells [5]. The opponents remain skeptical about this study and argue that there is still something violating medical ethics. Some scientists believe that this method is still immature, and it will take quite a long time for purpose of research and clinical treatment [6]. However, this does not mean that the study is not a good try. At least it provides a possible way to evade the ethical issues.

In the meanwhile, the progress of another new study shows a more promising application prospect. In 2006, Japanese scientists used retrovirus to insert four stem-cellrelated genes: Oct3/4, Sox $2, M y c$ and Klf4, into the genome of fibroblast cells in mouse. These cells showed the characteristics of embryonic stem cells. They called these cells induced pluripotent stem cells (iPSC) [7]. Since then, iPSC technology has achieved success for use in human beings, and such cells also exhibit the characteristics very similar to hES cells $[7,8]$. The generation process of iPS cells needs no embryo. Does this means that iPS cells can allow stem cell research to get rid of the binding of ethical issues? In fact, some researchers don't think that iPS cells can completely substitute hES cells. First, the current studies are still unable to make sure whether iPS cells are absolutely equivalent to early embryonic stem cells. Second, iPS cell research should be conducted simultaneously with hES cell research, otherwise it is impossible to assess the characteristics and research potential of iPS cells. Third, in some research fields, such as research on early human development, we can only use hES cells. Finally, the safety of iPS cells remains to be demonstrated. So when iPS cells are used in clinical applications, we should avoid abuse. 


\section{B. Ethical Issues about Donors}

Even though hESC research has gotten rid of the ethical controversy about embryo itself, researchers and doctors still can't arbitrarily dispose embryos. As embryos themselves can't make any decision, embryo or gamete donors will be the most important factors to be taken into consideration for embryo disposal. Some basic ethical principles should be followed in treatment of donors. Belmont report pointed out that the principle of respecting individuals is very important for donors.

The most important content of respecting individuals is to allow individuals to enjoy autonomy. Regardless of aborted embryos, in-vitro fertilization (IVF) surplus embryos or embryos created through the cell nuclear transfer technology, each source has the corresponding donor. Regardless of whether embryos or gametes are provided by donors to scientific institutions, this should be done voluntarily. Donors should be supplied sufficient information and fully understand the information before they agree to donate embryos. They should make a voluntary choice, i.e. informed consent.

First, it is very necessary to disclose the information to donors to prevent the use of embryos from other purposes which are unknown to donors. At present, part of embryos are deliberately created in the process of IVF only for research and therapy purposes, while this is completely unknown to donors. This kind of behavior not only violates the ethical requirements, but also will cause unnecessary harm to the health of embryo donors. Extraction of too many eggs will increase the risk of ovarian hyperstimulation syndrome (OHSS) [9]. Disclosure of information means that the donors should be not only informed of the whereabouts of embryos, but also be fully informed of the experimental process, the potential risks, the purpose of the experiment, the expected results and other contents. When necessary, the data on risk assessment and benefits should be also provided. Furthermore, if the downstream stem cell research will involve the disclosure of genetic information of the donors and other issues, it is particularly important to get informed consent. Researchers need to provide more details to donors, including for what kinds of research the stem cells derived from donated materials will be used, such as genetic modification of cells, large-scale genome sequencing and derivation of gametes from pluripotent stem cells for regeneration research [10]. Although the donation may be affected after acquiring such information, this is necessary for showing respect to the donors.

It is very important for donors to understand the information acquired. As most donors have limited biomedical knowledge, fully understanding the process and method of embryo disposal in experiments will help them know how to make choice. Therefore, medical workers should make efforts to ensure that donors have fully understood the information given. Only when donors make the decision to donate embryos rather than retain them or donate to other couples after they have understood the information clearly, the embryos can be used for scientific research or therapy.
Voluntary donation should be completely independent and free from any interference and force. If researchers and medical workers tempt owners of embryos to donate surplus embryos during the abortion or IVF treatment by providing payments, this also violates the principle of voluntariness. In such case, donors are subject to some interests or under pressure to make decisions, so they could not follow their real desire to make choices. In addition, high return means trading of embryos, which causes more serious ethical problems. It is legitimate to pay necessary remuneration such as document fee to donors, but such remunerations should not exceed a certain range.

As the behaviors discussed above aim to acquire embryos and extract hES cells for research purpose, this process involve not only the medical workers who implement IVF, also researchers play a more important role in this process and are even "principal conspirators". It is believed that researchers and medical workers are coconspirators in this process. Researchers make use of the actions implemented by others and so will not be directly or indirectly involved in the immoral behaviors [11]. We believe that, in solving this problem, the constraint to researchers should be strengthened, and this can be achieved through a rigorous examination to the projects of stem cell research, including their embryo sources and informed consent of donors.

The three principles, namely information, understanding and voluntariness, constitute the three elements of informed consent. In the process of practical application, researchers and medical workers should strictly follow the principle of informed consent, but actually they will encounter some complex situations. For example, donors are only under educated and can't understand the related information, or donors have another personal relationships with medical workers. In such case, more perfect detailed supervisory regulations are needed to ensure the effectiveness of informed consent. In general, in hESC research and therapy, we should fully respect the important participants - embryo donors, who are indispensable for the generation of those research results.

\section{Discussions on Public Policy CONCERning Stem Cell Research}

The ethical issue of stem cell research is of great concern to the public since it touches on the fundamental values of life, humanity and society. As different countries have different value judgment because of their different locality, culture and value, a number of ethical controversies have been produced. However, hESC research is so important to human beings that we can't give up its promising prospects simply because of its pending ethical controversy. The optimal choice is that we formulate appropriate ethical policies to bring the stem cell research under strict regulation, so it can continue to march on the right track.

At present, no unified principle and public policy have been formulated concerning hESC research internationally. The fact is that different public policies have been made by individual countries in light of their own respective 
circumstances. In spite of the difference in public policies among countries in terms of their strictness and completeness, their fundamental objective is to promote the research of hESC, ban or limit reproductive cloning and advocate the respect to human beings. In general, Germans hold an attitude of strict control for the protection of fertilized eggs and embryos. They have developed an Embryo Protection Law to give dignity and right to life of the embryos and prohibit preimplantation genetic diagnosis. Italy takes a more extreme attitude towards the issue of assisted reproduction. An Italian legislation concerning assisted preproduction, which launched in 2004, explicitly prohibits preimplantation genetic diagnosis for research purpose, artificial insemination, embryo freezing and extrajudicial preimplantation genetic diagnosis. The legislation also prohibits assisted reproduction of single women and stipulates that a maximum of three in vitro fertilized cells are allowed to be transferred to the ovaries when IVF assisted reproduction are used and they must be transferred at the same time. Once assisted reproductive technology is agreed by both husband and wife, it can't be revoked. Doctors, who violate the foregoing stipulation, will be prosecuted for their criminal liability and their medical qualification will be suspended. British policy permits cloning of early human embryos and extraction of stem cells from it for medical research. The British government has already granted a license valid for one year to the University of Newcastle, allowing it to carry on therapeutic cloning. However, the British government prohibits reproductive cloning and creating of human-animal hybrid embryos. The US policy on stem cell research is rather relaxed. The objective of the policy is to discourage them by regulating federal funding rather than absolutely ban them. Therefore, the controversy of the US on stem cell research lies in whether the federal funding can be used for financing stem cell research rather than just ban on it. From the Clinton's to the Obama's administration, the US has experienced a process from limiting federal funding for stem cell research to gradually relaxing strict control. Because of the gradual presentation of tremendous value and application prospects of stem cell research, the projects, in which only human embryo stem cell line established after 2001 is used, can be supported by federal funding.

For protection the embryo's moral status, different countries should think about their respective cultural and religious environments for formulating their policies. In a country with diversified religion and culture, there is no unified conception on the moral status of the preimplantation embryos among religious traditions. In this case, on one hand, decision-makers should adopt an inclusive approach to develop management guidelines by taking into account both personal beliefs and different religious beliefs; on the other hand, public policies should not be made in a bias towards one particular view of embryo ethics. The advantage of the United States in this regard is that in addition to federal funding, all states have developed their own government-funded policies in light of their respective circumstances. There are currently nine states prohibiting the use of in vitro embryos for research and other five states prohibiting government-funded hESC research, while the State of California supports hESC research. In such a way, the policies are made to a greater extent to adapt the cultural and religious environment of the country.

With regard to the protection of embryo donors, policies should reflect the principle of informed consent. The issues arising from the implementation of the policies should be detailed to ensure that each link is brought under a strict discipline and supervision. How to ensure that decisions of the donors on the donation of IVF surplus embryos are made under their informed consent and non-coercion conditions, remains a challenge for policy-makers. A few cases have showed that some institutions for stem cell research have tempted embryo donors to donate their embryos with a huge amount of money or tempted female staff working in the organization to donate their eggs. Such cases involve the sale of embryos or gametes, so it is a very serious moral issue. Therefore, it is necessary to develop strict policies to limit the relevant personnel. In general, couples who undergo IVF treatment should be informed the disposal choices of their surplus embryos prior to the treatment, which should include: destructing all remaining embryos after the IVF treatment is complete; freezing unused embryos for further use or donating to other couples; donating surplus unused embryos for research purposes. A second informed consent should be made at the end of treatment. Only those embryos of which the donation is for research purpose and is agreed by both husband and wife before and after treatment, can be used to derive stem cells. These principles for formulating policies of stem cell research have been accepted by a number of countries.

In China, relatively relaxed environment for stem cell research brings opportunity for stem cell research and treatment, but we still have to maintain a basic reverence for life, and give respect to embryos. Therefore, we should comply with corresponding procedures and guidelines in the acquisition and process of embryos. Qiu Renzong, an bioethicist has proposed that, for respect to the human embryo and disposal procedure of human embryo, it should include: human embryos used for the purpose of research must be in vitro; embryo used for research should not exceed 14 days; human embryo can only be used for the research which can't be executed without human embryos; embryo is not a commodity and it can't be traded; scientists should take necessary action to commemorate the contribution of the embryo; burial or cremation of an embryo should be conducted in a certain way and there should be a simple solemn ceremony [12]. With the continuous research progress in life science, new situations and problems will continue to emerge. Therefore, it is necessary to improve the existed policy in detail on the basis of fully investigation of new research developments and establish regulatory organization and advisory committee on national level to improve policy making system. For example, for "The Ethical Guiding Principle on Human Embryonic Stem Cell Research", which is promulgated jointly by the Ministry of Science and Technology and the Ministry of Health in 2003, we think, it should be 
complemented and improved on the basis of full deliberation, and specific provisions involving the review for the source of embryonic stem cells should be added especially.

The debate on embryonic stem cells will continue worldwide for a long time. However, with the deepening of the stem cell research and continuous adjustment of ethical policy of all countries concerned, the stem cell research will progress in twists and turns, and will eventually be applied in disease treatment in a deeper way.

\section{ACKNOWLEDGMENT}

The contents of this paper is based on the research achievements of the project "Study of Ethic Issues in Scientific Research and Its Policy Suggestion" (2011GXS4K078), which is supported by The National Soft Science Research Plan from the Ministry of Science and Technology of China.

\section{REFERENCES}

[1] Gearhart J, New potential for human embryonic stem cells, Science, 1998, 282(5391), pp. 1061-1062.

[2] Thomson J, Itskovitz-Eldor J, Shapiro S, et al., Embryonic stem cell lines derived from human blastocysts, Science, 1998, 282(5391), pp. 1145-1147.
[3] Ménard C, Hagège AA, Agbulut $\mathrm{O}$, et al., Transplantation of cardiaccommitted mouse embryonic stem cells to infarcted sheep myocardium: a preclinical study, Lancet, 2005, 366(9490), pp. 10051012.

[4] Stem Cell Research: Medical Progress with Responsibility, London, Department of Health, 2000, p. 58.

[5] Klimanskaya I, Chung Y, Becker S, et al., Derivation of human embryonic stem cells from single blastomeres, Nat Protoc, 2007, 2(8), pp. 1963-1972.

[6] Lok C, With a history of public blunders, can Advanced Cell Technology make embryonic stem-cell therapies a reality, nature, 2012, 481, pp. 130-133.

[7] Takahashi K, Yamanaka S, Induction of pluripotent stem cells from mouse embryonic and adult fibroblast cultures by defined factors, Cell, 2006, 126(4), pp. 663-676.

[8] Yu J, Vodyanik M, Smuga-Otto K, et al., Induced pluripotent stem cell lines derived from human somatic cells, Science, 2007, 318(5858), pp. 1917-1920.

[9] Giudice L, Santa E, Pool R, Assessing the Medical Risks of Human Oocyte Donation for Stem Cell Research, Washington, D.C., National Academies Press, 2007.

[10] Lo B, Parham L, Broom C, et al., Importing human pluripotent stem cell lines, Cell Stem Cell, 2009(4), pp. 115-12.

[11] Birnbacher D, Embryonic stem cell research and the argument of complicity, Ethics, Bioscience and Life, 2009(4), pp. 12-16.

[12] Zhai Xiaomei, Qiu Renzong. Introduction to Bioethics. Beijing: Tsinghua University Press, 2005, pp. 225- 256. 